\title{
The Application of Project-based Teaching in Information System Courses
}

\author{
Qing ZHOU* \\ China University of Petroleum-Beijing at Karamay, China \\ ${ }^{*}$ Corresponding author
}

Keywords: Project based teaching, Information system analysis and design, Unified modeling language.

\begin{abstract}
With the rapid development of information technology, it is necessary to adopt appropriate teaching models to cultivate students' self-learning ability and independent exploration capabilities. Therefore, in the information system curriculum, it is necessary to explore effective teaching methods and cultivate students' independent thinking skills. This paper discusses the characteristics of information system analysis and design courses in the core curriculum of software engineering specialty. It adopts project-based teaching method to cultivate students' professional skills and ability to solve problems.
\end{abstract}

\section{项目驱动式教学在信息系统课程中的实践}

\author{
周庆 ${ }^{*}$ \\ 中国石油大学（北京）-克拉玛依校区 计算机系，克拉玛依，中国 \\ “通讯作者
}

关键词：项目驱动式教学；信息系统分析与设计；UML

摘要: 为了适应信息技术的快速发展, 学生需要具有较强的自学能力和自主探索能力。因此, 在信息系统课程中, 探索有效的教学方法, 培养学生独立思考能力是十分必要的。本文探讨 了在软件工程专业核心课程中, 针对信息系统分析与设计课程的特点, 采用项目驱动式教学 法, 培养学生的专业技能和解决问题的能力。

\section{1. 引言}

21 世纪是信息时代，目前西方发达国家在信息科学领域处于领先地位，国内在软件工程、 信息管理与信息系统等专业的教学中, 应该吸收国外的先进教学成果, 突破传统教学模式的 局限，引入有效的教学方法，致力于提高学生的专业技能和解决问题的能力。

《信息系统分析与设计》作为信息系统开发的必要专业知识，在国内各高校软件工程专 业、信管专业均为重要的专业课程, 是软件工程、信管等专业的学生所需要的知识结构的重 要组成部分。通过这门课程的教学, 希望能够帮助学生系统地掌握对企业信息系统进行分析 与设计的方法，从而使学生具备参与各类信息系统项目建设的能力，为将来从事信息系统的 管理工作奠定基础。

为了提高该课程的可学性、可理解性, 许多学者都尝试通过探索课程的特点, 采取新的 教学方法来帮助学生掌握专业知识, 提高专业技能。目前, 国外的建构主义学习理论注重从 知识架构、思维方式等方面, 强调学习的主动性, 提倡情境性教学。而“项目驱动教学 (Project Based Teaching) 模式是一种建立在建构主义（Constructivism）理论基础上的教学方法。”[1] 
项目驱动教学采用项目开发模式, 强调以学生为中心组建项目开发团队, 教师充当指导者的

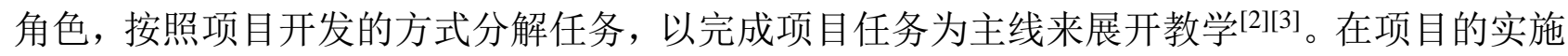
过程中, 按照信息系统开发的规范分析系统业务需求, 确定系统的功能模块, 并提供系统设 计方案, 指导学生去发现问题和解决问题, 帮助学生将所学专业知识融会贯通。与传统的教 学方法相比, 项目驱动法参照实际的项目开发模式, 组织学生团队作业, 充分调动学生的积 极性, 提高学生的学习兴趣和参与性, 营造一个良好互动的学习氛围。

\section{2. 课程特点}

《信息系统分析与设计》课程的理论性与实践性都较强, 传统的教学模式强调理论教学, 注重介绍目前主流的系统分析设计方法, 重点教授面向对象方法的概念理论、建模技术等内 容。

这门课程的特点, 首先是涉及知识面广, 以学生前面所修相关课程为基础, 包括数据库、 程序设计、管理信息系统等课程内容; 其次, 要求所学专业知识融会贯通, 本课程教学要求 学生掌握信息系统开发的面向对象分析方法、主流的面向对象建模技术, 提供信息系统的设 计方案, 要求学生将建模技术、数据库技术、编程技术等相关专业知识融会贯通, 运用到信 息系统分析设计的整个过程; 第三, 理论性与实践性都较强, 面向对象的系统分析设计理念 来源于面向对象编程, 由于本科生缺乏信息系统开发实践经验, 编程经验较少, 在理解面向 对象的系统分析设计方法和抽象概念方面，存在一定困难，相对难以掌握。

总之, 本课程从强调系统分析设计建模专业知识和系统开发能力这两个角度出发, 培养 学生的信息系统开发技能。引入项目驱动教学、以一个实际应用系统为背景, 详细剖析信息 系统分析设计方法的知识点, 包括UML（Unified Modeling Language）建模技术所涉及的各类 分析设计模型, 例如: 系统用例图、类图、活动图、状态图、顺序图、协作图、配置图、构 件图等。在教学过程中探索互动 (教师与学生) 、实践 (学习与运用) 等的恰当结合; 为学 生提供中英文的教学案例资料, 以及信息系统分析设计所采用的规格文档的中英文版, 让学 生们了解信息系统开发文档的中英文格式规范要求。通过对实际系统开发案例的分析探讨, 理解信息系统开发的过程与方法, 体现合作教学、合作学习、自主学习和探究学习等课程理 念。

\section{3. 学生的认识误区}

由于软件工程、信管专业是计算机技术和数据管理科学相结合的专业, 在专业课程设计 中不免存在着偏好问题, 有的学校偏重计算机技术, 有的学校偏重于管理。而对于学生来说 由于计算机技术类课程: 例如C语言、JAVA语言、数据结构等课程相对于项目管理类课程, 学生们在学习过程中会遇到时更多的困难, 因此会存在着一些认知误区。

\section{1 技术为王}

不少的学生认为互联网+时代, 技术为王。由于在学习过程中, 类似于C语言、JVAV语 言等程序类课程学习难度大, 掌握编程语言需要付出更多的努力才能考出好成绩。因此, 认 为软件工程等专业学习程序语言类课程是最重要的, 也愿意付出更多的时间来; 而对于“系统 分析与设计”这类课程, 偏重于理论知识, 认为实用性不强, 临近考试时死记硬背就能考出好 成绩，因此造成对于这门课程容易被学生忽略。

\section{2 轻视理论}

在重视计算机程序课程的同时, 学生们普遍存在着轻视理论课程学习的误区, 出于应试 教育的惯性, 对于理论课程的学习认为主要是记住原理, 背好名词解释, 就能在期末考试中 考出好成绩。至于所学的理论知识应该如何指导实践? 如何在项目开发中解决实际问题? 却 
是感触不深。理论与实践的脱节, 也是课程教学中会常常遇到的问题, 也与学生缺乏工作经 验, 缺乏对企业具体业务流程操作的了解有关。

\section{3 缺乏经验}

由于学生没有业务工作经验，对企业管理中存在的问题缺乏认知，不清楚哪些业务环节 需要通过计算机实现? 应该如何将业务处理过程有效地转化为计算机处理过程等; 同时又缺 少信息系统项目的开发实践, 这都使得学生在理解面向对象系统分析设计的理念、方法要点、 实施步骤等方面存在一定的困难。因此选择一个适合的项目来让学生实际操作, 就是任课教 师需要解决的问题。

\section{4. 项目案例的选择}

开展《信息系统分析与设计》课程教学实践, 由具有较好教学经验、能及时掌握本专业 最新研究成果的教师负责, 有利于在教学过程中及时引入国内外《信息系统分析与设计》课 程的先进成果和方法, 积极学习国内外先进的教学理念和教学方法, 采取理论教学和实验教 学相结合的方式, 充分利用现代教育手段和丰富的课程网络资源, 帮助学生更好地掌握《信 息系统分析与设计》的基本理论与实际应用。

此外, 本课程强调培养学生的动手能力和分析问题、解决问题的能力, 引入项目驱动教 学, 有助于学生更深入的理解信息系统分析与设计方法、相关理论知识。在项目案例的选择 上需要注意以下几个方面:

\section{1 行业特色}

因为学校办学方针强调石油行业背景, 学生就业首先面向石油行业。因此在项目的选择 上要考虑与石油业务管理相关的项目案例, 有利于帮助学生对石油行业相关业务进行了解, 便于学生毕业后能够迅速融入到实际工作中。

\section{2 难度适中}

因为课时量的限制，所选的项目不能太复杂规模太多，否则学生难以按时完成。但太简 单的话, 又达不到让学生掌握系统分析设计、解决实际问题的目的。这样的项目, 业务处理 过程清晰、系统需要明确、信息系统的功能模块数、数据表结构不超过 20 个。

\section{3 典型性}

所选择的项目是管理业务中普遍存在的系统需求, 但该项目的业务需求又具有一定特点; 所需完成的系统功能应包括一般系统的基本功能, 例如: 用户注册登录模块、用户授权管理、 单表录入查询模块、统计图形显示、系统日志管理等; 此外, 还应包括一些有特色的多维表 单的处理等。其次, 业务需求不能过于复杂专业, 但也不是一目了然, 需要在老师的启发下, 学生们能够理解并掌握业务处理要点; 同时, 能够让学生们将来在工作中遇到同样问题时知 道如何去分析解决。

为了达到以上目的, 任课老师需结合实际工作经验, 精心挑选, 所选项目既要与学生的 理解能力相适应, 又要有实际业务背景。经过综合比较, 最终选定了某石油管道供应商的“加 油站管道信息备案系统”作为项目实施案例; 对该项目案例中的原始材料进行归纳总结, 为 学生提供原始的业务表单、引导学生了解实际业务情况, 同时又对项目系统需求内容进行加 工整理、根据教学要求进行调整, 便于学生理解掌握。

\section{5. 项目实践}

注重使用先进的教学手段, 本课程重点在于实践教学, 改革学生考核方法, 注重实践环 节中学生的任务完成情况。采用问题探讨或项目开发等互动式教学方法, 要求学生们结合石 
油行业的实际应用, 以“加油站管道信息备案系统”为项目开发课题，上机实习。以“加油站管 道信息备案系统” 为背景, 按照实际信息系统的开发要求, 组建开发团队, 以小组长负责制分 组、分阶段完成一个简单的基于WEB的“加油站管道信息备案系统”的开发。

首先从系统调研开始, 引导学生调查现有系统运行状况, 分析用户可能的系统需求, 采 用用例模型完成系统功能需求描述 (图1)、以及系统业务流程分析; 在此基础上引导学生分 组讨论, 完成系统的详细设计, 包括: 数据库表结构设计、输入/输出设计、界面设计（图2) 等。指导学生们完成系统的分析设计报告 (老师参与各小组的讨论, 回答学生的疑问、引导 学生完成系统的详细分析设计) 、并且要求各小组分别上讲台汇报每个阶段的工作成果, 接 受老师对项目各阶段完成情况的质询, 给予工作成绩评比。

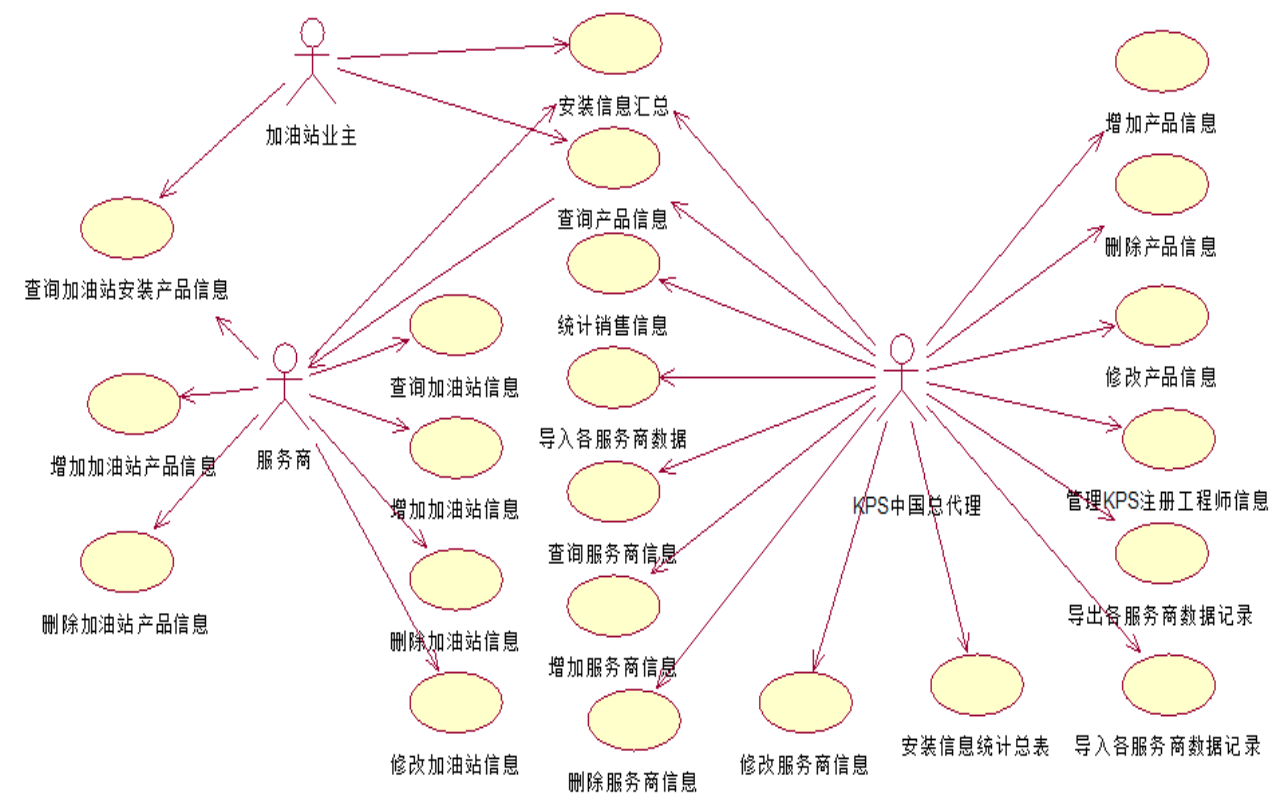

图1 系统用例
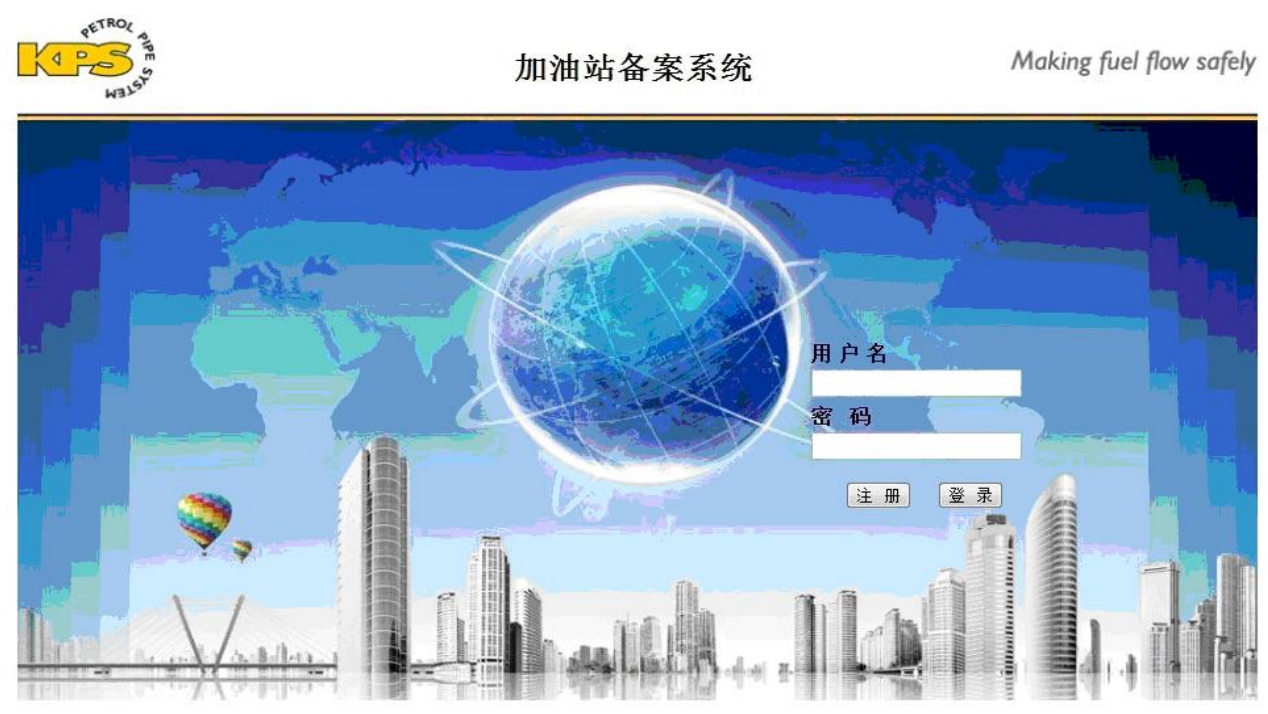

图2 系统登录界面

同时指导学生进行信息系统开发环境的配置工作，包括网络操作系统的配置、WEB服务 器和数据库服务器的配置。

为了帮助学生能够迅速掌握基于WEB的程序编写, 老师向学生提供一个“用户注册”的JSP 小程序作为入门例子, 讲解在WEB环境下如何进行数据库连接操作, 以及JSP动态网页开发 技术, 让学生们理解WEB环境下的程序编写技术。 
指导学生们的动态网页的编程, 实现基于WEB的“加油站管道信息备案系统”的部分系统 功能，完成包括“服务商管理、地区代码、加油站管理、用户类型、产品类型、产品信息管理、 计量单位代码、加油站安装信息管理、KPS注册工程师信息管理、加油站所属公司的代码、 系统用户管理、系统授权管理、系统日志管理、安装信息汇总统计”等多个功能模块的程序编 写任务。让学生熟悉一个实际WEB应用系统的开发过程, 掌握相关开发技术, 从而提高学生 的动手能力和实践能力, 为学生将来的学习与工作实践奠定坚实的理论与应用基础。

\section{6. 结束语}

本课程教学实践主要采用项目驱动的教学方式, 引导学生采用面向对象的系统分析设计 方法开发“加油站管道信息备案系统”项目。项目驱动式教学需要任课教师具备丰富的教学经 验和管理信息系统的开发经验, 老师在整个教学过程中需要起到组织、指导、启发学生积极 思考的作用, 能与学生保持良好的互动, 即时为学生答疑解惑。通过项目驱动式教学实践, 尝试建设以提高素质和能力为中心的优化课程体系, 积极更新教育思想观念, 结合案例教学 方法、更新教学手段和教学管理。

\section{致谢}

本文由中国石油大学（北京）-克拉玛依校区研究基金 (RCYJ2017B-03-002) 支持。

\section{References}

[1] Yabin Xu, Weizhen Zhou, Yunmei Shi, (2011). Research and Practice of Project-Based Teaching, Journal of Liaoning University of Technology (Social Science Edition). 13(3), 125-127.

[2] Tao SONG, Yu-lin WANG, Ming-fu ZHAO, Nian-bing ZHONG. et al. (2017). Exploration of the Teaching Mode in Colleges under the Project Driven Mechanism, Education Teaching Forum. 2017 (26), 141-142.

[3] Zhu Bin, Cao Manxiang,Xing Yingshou,Huang Jiangbo, (2015) The Application of Project-driven Teaching Mode in Practice Teaching, Science \& Technology Information. 2015(36), 220-222.

[4] SHAO Fang, (2018). A Project-based Minilecture Teaching Pattern, Journal of Jilin Engineering Normal University. 2018(1), 65-67

[5] Straub, J (Straub, Jeremy); Kerlin, S (Kerlin, Scott); Whalen, D (Whalen, David), (2017). Teaching Software Project Management Using Project Based Learning (PBL) and Group Projects, 2017 IEEE INTERNATIONAL CONFERENCE ON ELECTRO INFORMATION TECHNOLOGY (EIT), pp16-21, 2017. 